Research Paper

\title{
High Expression Levels of ACTNI and ACTN3 Indicate Unfavorable Prognosis in Acute Myeloid Leukemia
}

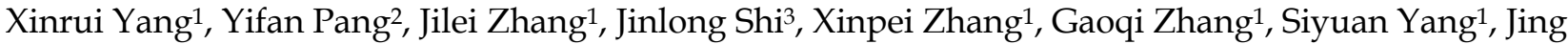 \\ Wang ${ }^{1}$, Kai Hu${ }^{1}$, Jijun Wang1, Hongmei Jing ${ }^{1}$, Xiaoyan $\mathrm{Ke}^{1 凶}$, Lin Fu ${ }^{\circledR}$ \\ 1. Department of Hematology and Lymphoma Research Center, Peking University, Third Hospital, Beijing, 100191, China. \\ 2. Department of Medicine, William Beaumont Hospital, Royal Oak, MI 48073, USA. \\ 3. Department of Biomedical Engineering, Chinese PLA General Hospital, Beijing, 100853, China.
}

$\square$ Corresponding authors: Lin Fu; Department of Hematology and Lymphoma Research Center, Peking University, Third Hospital, 49 Huayuan North Road, Beijing 100191, China. Tel: +86-10- 82267651; Fax: +86-10-82267651; Email: fulin022@126.com; or Xiaoyan Ke; Department of Hematology and Lymphoma Research Center, Peking University, Third Hospital, 49 Huayuan North Road, Beijing 100191, China. Tel: +86-10- 82266780; Fax: +86-10-82266780; Email: xykbysy@163.com.

(c) The author(s). This is an open access article distributed under the terms of the Creative Commons Attribution License (https://creativecommons.org/licenses/by/4.0/). See http://ivyspring.com/terms for full terms and conditions.

Received: 2018.11.24; Accepted: 2019.05.14; Published: 2019.07.10

\begin{abstract}
Background: Actinins are major cytoskeletal proteins that mediate sarcomere function, and they also have important non-muscle functions such as regulating cytokinesis, cell adhesion and migration. There are four isoforms of actinins in mammals (ACTN1-4). Recently, the relationship between actinins and cancer has been discovered in many types of malignancy, yet their prognostic significance in acute myeloid leukemia $(A M L)$ remains unclear.

Methods: We collected data of 155 de novo AML patients from The Cancer Genome Atlas (TCGA) database; 85 patients received chemotherapy only and 70 patients underwent allogeneic hematopoietic stem cell transplantation (allo-HSCT). We divided each treatment groups into sub-groups based on the median expression levels of ACTNI-4.

Results: Survival analysis showed that in the chemotherapy-only group, high ACTNI and ACTN3 expression were associated with shorter event-free survival (EFS) and overall survival (OS) $(p<0.01)$. Multivariate analysis suggested that high expression of ACTNI and ACTN3 $(p<0.05)$ were independent poor prognostic factors. In the allo-HSCT group, ACTNI-4 expression had no impact on survival.

Conclusions: Our study suggested that high expression levels of ACTNI and ACTN3 adversely affected the survival of AML patients, but their harmful impact could be overcome by allo-HSCT.
\end{abstract}

Key words: acute myeloid leukemia; ACTN1; ACTN3; prognosis

\section{Background}

Acute myeloid leukemia (AML) is a highly heterogeneous and aggressive malignancy of the undifferentiated or partially-differentiated bone marrow myeloid stem cells and progenitor cells ${ }^{1}$. In the past decade, next generation sequencing (NGS) technique has been widely used and became a powerful tool in AML research, greatly improving our understanding of the genetic basis of the disease. Many prognostic biomarkers were found with the help of NGS. For example, NPM1 mutation and biallelic CEBPA mutations are favorable factors, associating with longer event-free survival (EFS) and overall survival (OS). On the other hand, the present of FLT3-ITD and MLL-PTD, mutations in DNMT3A, RUNX1, TET2, and KRAS are predictors for poor outcomes in AML patients-4. In addition, aberrant epigenetic modification, i.e., dysregulated expression levels of certain genes, may also influence the prognosis.

Actinins are a group of cytoskeletal molecules that belongs to the actin filament cross-linking proteins $^{5}$. There are four actinin isoforms in mammals, namely ACTN1-4. ACTN1 and ACTN4 are universally expressed in most tissues and cell types. 
ACTN2 are mainly expressed in the myocardium, skeletal muscle and brain. ACTN3 mostly appear in the skeletal muscle. In muscle cells, actinins link the adjacent sarcomeres together through thin filaments, to coordinate muscle contraction. In non-muscle cells, actinins also exhibit a myriad of functions ${ }^{6}$. Actinins participate in cytokinesis by balancing the contraction of myosin II, forming a contractile ring with the latter to eventually divide the mother cell in two. Actinins can format and disassemble cell-matrix adhesion through the activation of phosphoinositide 3- kinase (PI3K), or build different cell-cell adhesions by working with integrins and intercellular adhesion molecules (ICAMs). They are also indispensable for endocytosis and exocytosis, which are essential biological processes for neurons and synapses. ACTN4 has recently been found to be a transcriptional regulator.

Since ACTN4's prognostic value in breast cancer was established, many works have been done to investigate the role of actinins in cancer prognostication and tumorigenesis ${ }^{7}$. Increased expression of ACTN4 has been associated with poor prognosis in ovarian cancer, colorectal cancer and acute lymphoblastic leukemia ${ }^{8-10}$. ACTN1 is crucial for glioma cell motility, and it also plays an important role in lung adenocarcinoma ${ }^{11,12}$. There are also studies pointing out the interactions between ACTN2 and ACTN3 with the parafibromin tumor suppressor protein, one of the proteins that is involved in the hypermethylation and suppression of many oncogenes ${ }^{13}$.

However, the impacts of ACTN1-4 on the clinical and biological features of AML, as well as their prognostic value, remain unclear. Herein, we analyzed the relationship between the expression levels of ACTN1-4 and the outcomes of AML patients, hoping to guide future research in these areas.

\section{Methods}

\section{Patients}

We screened for de novo AML patients with ACTN1-4 expression data at diagnosis from The Cancer Genome Atlas (TCGA) database. A total of 155 patients had available data for analysis. Among them, 85 patients received only chemotherapy (chemotherapy-only group) and 70 patients underwent allogeneic hematopoietic stem cell transplantation (allo-HSCT, allo-HSCT group). Baseline demographic, laboratory and genetic data were downloaded from the TCGA public website. Gene expression profiling was performed on the Affymetrix U133 plus 2 platform. The Clinical endpoints were event-free survival (EFS) and overall survival (OS). EFS is the time from diagnosis to removal from the study due to death, relapse, failure to achieve complete remission (CR), or censored at the last follow-up. OS is the time from diagnosis to death from any cause or censored at the last follow-up. Written informed consent was obtained from all patients, and the database was approved by the Human Research Ethics Committee of Washington University.

\section{Statistical analysis}

The clinical and biological characteristics of patients were summarized by descriptive statistics. Numerical data was compared using the Mann-Whitney $U$ test and categorical data was compared using the Chi-square test. Survival was depicted by the Kaplan-Meier method and compared by the log-rank test. Cox proportional hazard models were constructed for multivariate analysis in search of independent factors that influenced survival. Hazard ratios were presented with $95 \%$ confidence intervals. A two-sided P-value $<0.05$ was considered statistically significant for all statistical analyses. All statistical analyses were performed by SPSS Version 20.0 software.

\section{Results}

\section{Comparison of EFS and OS between different expression levels of ACTNI-4}

We divided the chemotherapy-only group $(n=85)$ and allo-HSCT group $(n=70)$ patients into high and low-expression subgroups by their median ACTN1-4 expression levels at diagnosis. ACTN1-4 expression levels $\geq$ median was defined as high expression of the respective gene; others were defined as low expression. Kaplan-Meier method and the log-rank test were used for the analysis of EFS and OS between the high and low expression groups of each ACTN (Table 1A and 1B). In the chemotherapy-only group, patients with high expression levels of ACTN1 and ACTN3 had significantly shorter EFS ( $\mathrm{p}=0.002$, 0.006 , respectively) and OS ( $\mathrm{p}=0.003,0.006$, respectively), as shown in Figure 1A-D. ACTN2 was also influential $(\mathrm{p}=0.025$ for $E F S, p=0.039$ for $O S)$. However, ACTN4 had no effect on EFS and OS. There was no statistical significance in survival between each ACTN high and low-expression subgroups in the allo-HSCT group.

\section{Association of clinical and biological characteristics with ACTNI or ACTN3 expression levels}

As demonstrated above, ACTN1 and ACTN3 had more significant impact on EFS and OS than ACTN2 and ACTN4, hence we analyzed the association 
between the clinical, biological features and the expression levels of ACTN1 (high vs. low) and ACTN3 (high vs. low) in the entire cohort $(\mathrm{n}=155$, Table 2$)$. High ACTN1 expression was associate with more patients over age $60(p=0.037)$, lower WBC counts $(p=0.010)$, fewer patients with FAB-M4 $(p=0.032)$, more patients with complex karyotype $(p=0.022)$ or poor-risk cytogenetics $(\mathrm{p}=0.001)$. Patient with higher ACTN1 also had higher frequencies in TP53 $(\mathrm{p}=0.005)$ and WT1 $(\mathrm{p}=0.018)$. High ACTN3 expression was associate with more patients $>60$ years old, lower WBC counts, fewer patients with FAB-M4 subtype and more with FAB-M0 ( $\mathrm{p}=0.002, \mathrm{p}=0.002, \mathrm{p}=0.018$, and $\mathrm{p}=0.001$, respectively). Similar to ACTN1, patients with high ACTN3 expression also tended to have complex karyotype and poor-risk cytogenetics (both $\mathrm{p}=0.000)$. Fewer FLT3 mutations $(\mathrm{p}=0.002)$, more frequent IDH1/2 mutations $(\mathrm{p}=0.049)$ and TP53 mutations $(p=0.005)$ were found in high ACTN3 expression patients.

Table 1A. Comparison of EFS and OS between high and low expression levels of ACTNI-4 (Chemotherapy-only, $\mathrm{n}=85$ )

\begin{tabular}{lllll}
\hline Variables & EFS & \multicolumn{3}{l}{ OS } \\
\cline { 2 - 5 } & $\chi^{2}$ & $P$-value & $\chi^{2}$ & $P$-value \\
\hline ACTN1 (high vs. low) & 9.331 & 0.002 & 8.756 & 0.003 \\
ACTN2 (high vs. low) & 5.051 & 0.025 & 4.251 & 0.039 \\
ACTN3 (high vs. low) & 7.542 & 0.006 & 7.571 & 0.006 \\
ACTN4 (high vs. low) & 0.149 & 0.700 & 0.049 & 0.825 \\
\hline
\end{tabular}

Abbreviations: EFS, event-free survival; OS, overall survival.
Table 1B. Comparison of EFS and OS between high and low expression levels of ACTNI-4 (HSCT, $\mathrm{n}=70)$

\begin{tabular}{lllll}
\hline Variables & EFS & & OS \\
\cline { 2 - 5 } & $\chi^{2}$ & $P$-value & $\chi^{2}$ & $P$-value \\
\hline ACTN1 (high vs. low) & 0.521 & 0.470 & 0.284 & 0.594 \\
ACTN2 (high vs. low) & 0.042 & 0.838 & 0.164 & 0.685 \\
ACTN3 (high vs. low) & 0.012 & 0.915 & 0.845 & 0.385 \\
ACTN4 (high vs. low) & 0.106 & 0.744 & 0.096 & 0.757 \\
\hline
\end{tabular}

Abbreviations: EFS, event-free survival; OS, overall survival.

\section{Prognostic impact of ACTNI and ACTN3 expression in AML patients}

Kaplan-Meier survival curves suggested that in the chemotherapy-only group, AML patients with high expressions of either ACTN1 or ACTN3 had shorter EFS and OS compared with patients with low expressions ( $p=0.002$ for EFS, $p=0.003$ for OS, Figure $2 \mathrm{~A}$ and $2 \mathrm{~B}$ ). However, no significant difference was found when doing similar comparison in the allo-HSCT group ( $p>0.05$, Figure $3 \mathrm{~A}$ and $3 \mathrm{~B}$ ).

Multivariate analysis was implemented to evaluate the prognostic value of clinical and biological variables in the chemotherapy-only group in order to avoid the influence of allo-HSCT. The expression levels of ACTN1, ACTN2 and ACTN3 (high vs. low), age $(\geq 60$ vs. $<60$ years), WBC count ( $\geq 15$ vs. $<15 \times 109 / \mathrm{L}$ ), FLT3-ITD (positive vs. negative) and other common AML mutations with relatively high frequency in this study (NPM1, DNMT3A, IDH1/2, RUNX1, TET2 and NRAS/KRAS; mutated vs. wild
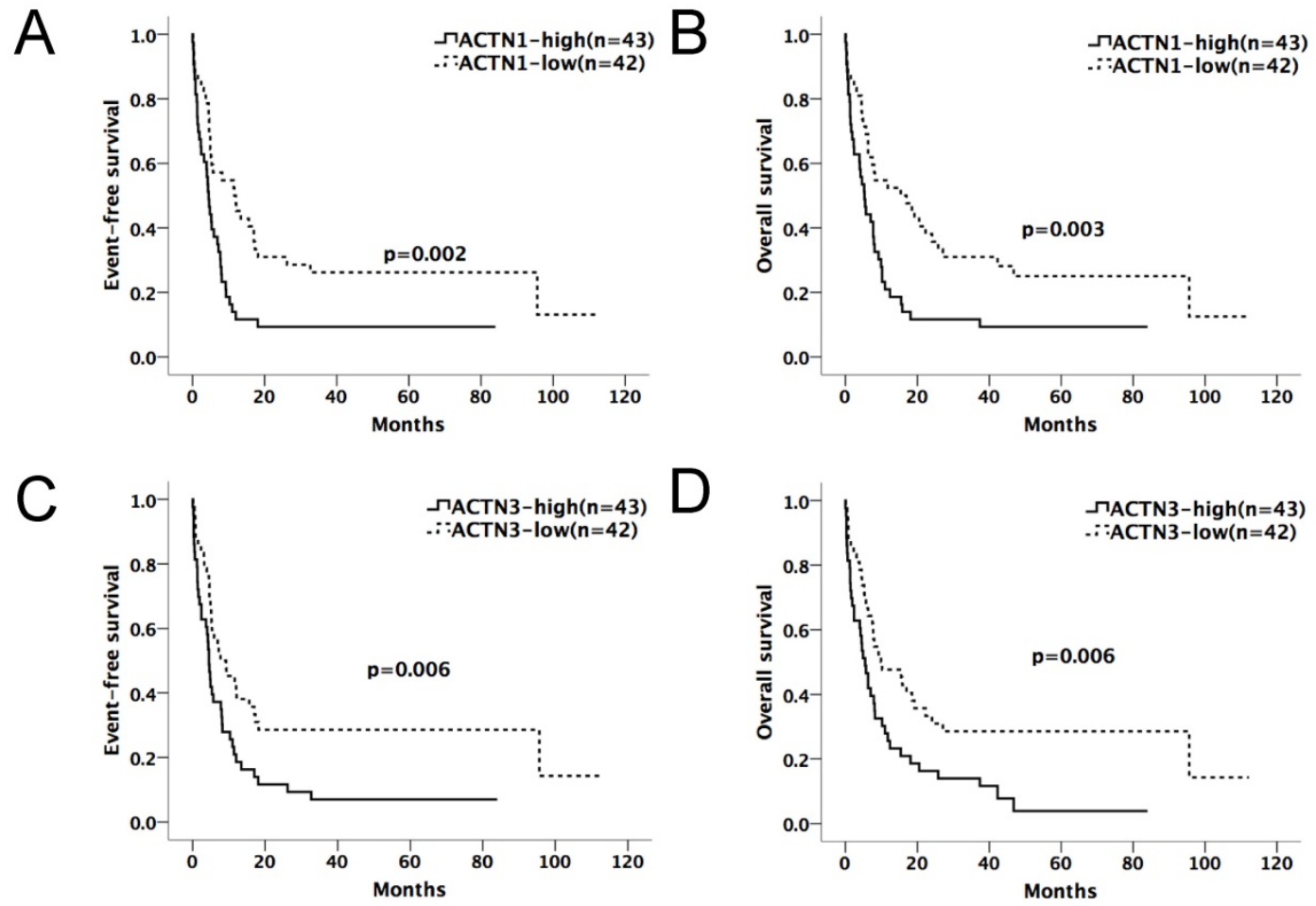

Figure 1. Expression levels of $A C T N I / A C T N 3$ and patients' outcome in the chemotherapy-only group $(\mathbf{n}=\mathbf{8 5})$. Patients with high expression levels of ACTN1 and ACTN3 had significantly shorter EFS ( $p=0.002,0.006$, respectively, Figures $1 \mathrm{~A}$ and $1 \mathrm{C})$ and $\mathrm{OS}(\mathrm{p}=0.003,0.006$, respectively, Figures $1 \mathrm{~B}$ and ID). 
type), were selected to construct the Cox regression model (Table 3). High expression levels of ACTN1 $(\mathrm{p}=0.007$ for EFS, $\mathrm{p}=0.021$ for OS) and ACTN3 $(p=0.048$ for EFS, $p=0.018$ for OS), older age $(p=0.000$ for EFS, $p=0.002$ for OS) independently contributed to poor prognosis.

Table 2. Clinical and molecular characteristics of patients with high or low ACTNI and ACTN3 expression levels

\begin{tabular}{|c|c|c|c|c|c|c|}
\hline \multirow[t]{2}{*}{ Characteristics } & \multicolumn{3}{|l|}{ ACTN1 } & \multicolumn{3}{|l|}{ ACTN3 } \\
\hline & High $(n=78)$ & Low $(n=77)$ & $P$ & High $(n=78)$ & Low $(n=77)$ & $P$ \\
\hline Age/years, median (range) & $61(18-81)$ & $57(22-88)$ & $0.126^{*}$ & $62.5(21-88)$ & $56(18-82)$ & $0.007^{*}$ \\
\hline Age group/n (\%) & & & $0.037 \S$ & & & $0.002^{\mathrm{s}}$ \\
\hline$<60$ years & $32(41.0)$ & $45(58.4)$ & & $29(37.2)$ & $48(62.3)$ & \\
\hline$\geq 60$ years & $46(59.0)$ & $32(41.6)$ & & $49(62.8)$ & $29(37.7)$ & \\
\hline Gender/n (\%) & & & $0.335 \S$ & & & $0.108^{\mathrm{s}}$ \\
\hline Male & $46(59.0)$ & $39(50.6)$ & & $48(61.5)$ & $37(48.1)$ & \\
\hline Female & $32(41.0)$ & $38(49.4)$ & & $30(38.5)$ & $40(51.9)$ & \\
\hline Race/n (\%) & & & $0.588 s$ & & & $0.275 \mathrm{~s}$ \\
\hline Caucasian & $59(75.6)$ & $55(71.4)$ & & $54(69.2)$ & $60(77.9)$ & \\
\hline Others & $19(24.4)$ & $22(28.6)$ & & $24(30.8)$ & $17(22.1)$ & \\
\hline WBC $/ \times 10^{9} / \mathrm{L}$, median (range) & $12.7(0.6-171.9)$ & $33.2(1.0-297.4)$ & $0.010^{*}$ & $11.1(0.6-297.4)$ & $33.2(1.2-223.8)$ & $0.002^{*}$ \\
\hline BM blast/\%, median (range) & $70.0(30-100)$ & $73(33-99)$ & $0.427^{\star}$ & $69(30-99)$ & $72(32-100)$ & $0.893^{*}$ \\
\hline PB blast/\%, median (range) & $32(0-97)$ & $48(0-98)$ & $0.076^{*}$ & $32(0-98)$ & $47(0-97)$ & $0.349^{*}$ \\
\hline \multicolumn{7}{|l|}{ FAB subtypes/n (\%) } \\
\hline M0 & $6(7.7)$ & $10(13.0)$ & $0.304 s$ & $14(17.9)$ & $2(2.6)$ & $0.001 \mathrm{~s}$ \\
\hline M1 & $24(30.8)$ & $19(24.7)$ & $0.473 s$ & $21(26.9)$ & $22(28.6)$ & $1.000 \mathrm{~s}$ \\
\hline M2 & $23(29.5)$ & $16(20.8)$ & $0.266 \S$ & $18(23.1)$ & $21(27.3)$ & $0.711 \mathrm{~s}$ \\
\hline M4 & $11(14.1)$ & $22(28.6)$ & $0.032^{\S}$ & $10(12.8)$ & $23(29.9)$ & $0.018^{\mathrm{s}}$ \\
\hline M5 & $10(12.8)$ & $7(9.1)$ & $0.608^{\S}$ & $9(11.5)$ & $8(10.4)$ & $0.803^{8}$ \\
\hline M6 & $1(1.3)$ & $1(1.3)$ & $1.000 \S$ & $2(2.6)$ & $0(0.0)$ & $0.245 \mathrm{~s}$ \\
\hline M7 & $2(2.6)$ & $1(1.3)$ & $1.000 \mathrm{~s}$ & $2(2.6)$ & $1(1.3)$ & $0.620 \mathrm{~s}$ \\
\hline No date & $1(1.3)$ & $1(1.3)$ & & $2(2.6)$ & $0(0.0)$ & \\
\hline \multicolumn{7}{|l|}{ Karyotype/n (\%) } \\
\hline Normal & $36(46.2)$ & $37(48.1)$ & $0.871^{\mathrm{s}}$ & $31(39.7)$ & $42(54.5)$ & 0.108 \\
\hline Complex & $17(21.8)$ & $6(7.8)$ & $0.022^{s}$ & $20(25.6)$ & $3(3.9)$ & $0.000^{\mathrm{s}}$ \\
\hline 8 Trisomy & $5(6.4)$ & $2(2.6)$ & $0.442 s$ & $6(7.7)$ & $1(1.3)$ & $0.062^{\mathrm{s}}$ \\
\hline $\operatorname{inv}(16) / C B F \beta-M Y H 11$ & $1(1.3)$ & $10(13.0)$ & $0.004 s$ & $0(0.0)$ & $11(14.3)$ & $0.001 \mathrm{~s}$ \\
\hline $11 \mathrm{q} 23 / \mathrm{MLL}$ & $5(6.4)$ & $1(1.3)$ & $0.210 \mathrm{~s}$ & $1(1.3)$ & $5(6.5)$ & $0.210 \mathrm{~s}$ \\
\hline$-7 / 7 q-$ & $5(6.4)$ & $1(1.3)$ & $0.210 \S$ & $6(7.7)$ & $0(0.0)$ & $0.013 \mathrm{~s}$ \\
\hline $\mathrm{t}(9 ; 22) / \mathrm{BCR}-\mathrm{ABL} 1$ & $2(2.6)$ & $1(1.3)$ & $1.000 \mathrm{~s}$ & $2(2.6)$ & $1(1.3)$ & $0.618^{\mathrm{s}}$ \\
\hline $\mathrm{t}(8 ; 21) /$ RUNX1-RUNX1T1 & $1(1.3)$ & $6(7.8)$ & $0.062 s$ & $1(1.3)$ & $6(7.8)$ & $0.117 \mathrm{~s}$ \\
\hline Others & $5(6.4)$ & $11(14.3)$ & $0.118 \S$ & $8(10.3)$ & $8(10.4)$ & $1.000 \mathrm{~s}$ \\
\hline No date & $1(1.3)$ & $2(2.6)$ & & $3(3.8)$ & $0(0.0)$ & \\
\hline \multicolumn{7}{|l|}{ Risk/n (\%) } \\
\hline Good & $2(2.6)$ & $16(20.8)$ & $0.000 \mathrm{~s}$ & $1(1.3)$ & $17(22.1)$ & $0.000 \mathrm{~s}$ \\
\hline Intermediate & $45(57.7)$ & $48(62.3)$ & $0.509 \S$ & $43(55.1)$ & $50(64.9)$ & $0.406^{\mathrm{s}}$ \\
\hline Poor & $30(38.5)$ & $11(14.3)$ & $0.001^{\S}$ & $31(39.7)$ & $10(13.0)$ & $0.000 \mathrm{~s}$ \\
\hline No date & $1(1.3)$ & $2(2.6)$ & & $3(3.8)$ & $0(0.0)$ & \\
\hline$M L L-P T D$ & & & $0.167 \S$ & & & $0.495 \mathrm{~s}$ \\
\hline Presence & $7(9.0)$ & $2(2.6)$ & & $6(7.7)$ & $3(3.9)$ & \\
\hline Absence & $71(91.0)$ & 75 (97.4) & & $72(92.3)$ & $74(96.1)$ & \\
\hline FLT3/n (\%) & & & $0.474 \mathrm{~s}$ & & & $0.002^{\mathrm{s}}$ \\
\hline FLT3-ITD & $17(21.8)$ & $14(18.2)$ & & $10(12.8)$ & $21(27.3)$ & \\
\hline FLT3-TKD & $7(9.0)$ & $5(6.5)$ & & $3(3.8)$ & $9(11.7)$ & \\
\hline Wild type & $54(69.2)$ & $58(75.3)$ & & $65(83.3)$ & $47(61.0)$ & \\
\hline NPM1/n (\%) & & & $0.599 s$ & & & $0.599 \mathrm{~s}$ \\
\hline Mutation & $21(26.9)$ & $24(31.2)$ & & $21(26.9)$ & $24(31.2)$ & \\
\hline Wild type & $57(73.1)$ & $53(68.8)$ & & $57(73.1)$ & $53(68.8)$ & \\
\hline DNMT3A/n (\%) & & & $1.000 \mathrm{~s}$ & & & $0.583 \mathrm{~s}$ \\
\hline Mutation & $20(25.6)$ & $20(26.0)$ & & $22(28.2)$ & $18(23.4)$ & \\
\hline Wild type & $58(74.4)$ & $57(74.0)$ & & $56(71.8)$ & $59(76.6)$ & \\
\hline IDH1/IDH2/n (\%) & & & $0.846 \S$ & & & $0.049 \mathrm{~s}$ \\
\hline Mutation & $16(20.5)$ & $17(22.1)$ & & $22(28.2)$ & $11(14.3)$ & \\
\hline Wild type & $62(79.5)$ & $60(77.9)$ & & $56(71.8)$ & $66(85.7)$ & \\
\hline RUNX1/n (\%) & & & $0.121^{\mathrm{s}}$ & & & $0.429 \mathrm{~s}$ \\
\hline Mutation & $5(6.4)$ & $11(14.3)$ & & $10(12.8)$ & $6(7.8)$ & \\
\hline Wild type & $73(93.6)$ & $66(85.7)$ & & $68(87.2)$ & $71(92.2)$ & \\
\hline TET2/n (\%) & & & $0.588 \S$ & & & $0.588 \mathrm{~s}$ \\
\hline Mutation & $9(11.5)$ & $6(7.8)$ & & $9(11.5)$ & $6(7.8)$ & \\
\hline Wild type & $69(88.5)$ & $71(92.2)$ & & $69(88.5)$ & $71(92.2)$ & \\
\hline TP53/n (\%) & & & $0.005 \S$ & & & $0.001 \mathrm{~s}$ \\
\hline Mutation & $13(16.7)$ & $2(2.6)$ & & $14(17.9)$ & $1(1.3)$ & \\
\hline Wild type & $65(83.3)$ & $75(97.4)$ & & $64(82.1)$ & $76(98.7)$ & \\
\hline NRAS/KRAS/n (\%) & & & $0.811^{\mathrm{s}}$ & & & $1.000^{\mathrm{s}}$ \\
\hline Mutation & $9(11.5)$ & $10(13.0)$ & & $10(12.8)$ & $9(11.7)$ & \\
\hline
\end{tabular}




\begin{tabular}{|c|c|c|c|c|c|c|}
\hline \multirow[t]{2}{*}{ Characteristics } & \multicolumn{3}{|l|}{ ACTN1 } & \multicolumn{3}{|l|}{ ACTN3 } \\
\hline & High $(n=78)$ & Low $(n=77)$ & $P$ & High $(\mathrm{n}=78)$ & Low $(n=77)$ & $P$ \\
\hline Wild type & $69(88.5)$ & $67(87.0)$ & & $68(87.2)$ & $68(88.3)$ & \\
\hline CEBPA/n (\%) & & & $0.765^{\S}$ & & & $0.368 \mathrm{~s}$ \\
\hline Single Mutation & $5(6.4)$ & $3(3.9)$ & & $4(5.1)$ & $4(5.2)$ & \\
\hline Double Mutation & $0(0.0)$ & $3(3.9)$ & & $0(0.0)$ & $3(3.9)$ & \\
\hline Wild type & $73(93.6)$ & $71(92.2)$ & & $74(94.9)$ & $70(90.9)$ & \\
\hline$W T 1 / \mathrm{n}(\%)$ & & & $0.018 \S$ & & & $0.534 \mathrm{~s}$ \\
\hline Mutation & $9(11.5)$ & $1(1.3)$ & & $4(5.1)$ & $6(7.8)$ & \\
\hline Wild type & $69(88.5)$ & $76(98.7)$ & & $74(94.9)$ & $71(92.2)$ & \\
\hline PTPN11/n (\%) & & & $0.719 \S$ & & & $0.495 \mathrm{~s}$ \\
\hline Mutation & $5(6.4)$ & $3(3.9)$ & & $3(3.8)$ & $5(6.5)$ & \\
\hline Wild type & 73 (93.6) & $74(96.1)$ & & $75(96.2)$ & $72(93.5)$ & \\
\hline Relapse/n (\%) & & & $0.265^{\S}$ & & & $0.426^{\mathrm{s}}$ \\
\hline Yes & $36(46.2)$ & $42(54.5)$ & & $37(47.4)$ & $41(53.2)$ & \\
\hline No & $42(53.8)$ & $34(44.2)$ & & $41(52.6)$ & $35(45.5)$ & \\
\hline No date & $0(0.0)$ & $1(1.3)$ & & $0(0.0)$ & $1(1.3)$ & \\
\hline
\end{tabular}

Abbreviations: WBC: white blood cell; BM: bone marrow; PB: peripheral blood; FAB: French American British.

‘*' denotes Mann-Whitney $U$ test; ' $\S$ ' denotes chi-square test.
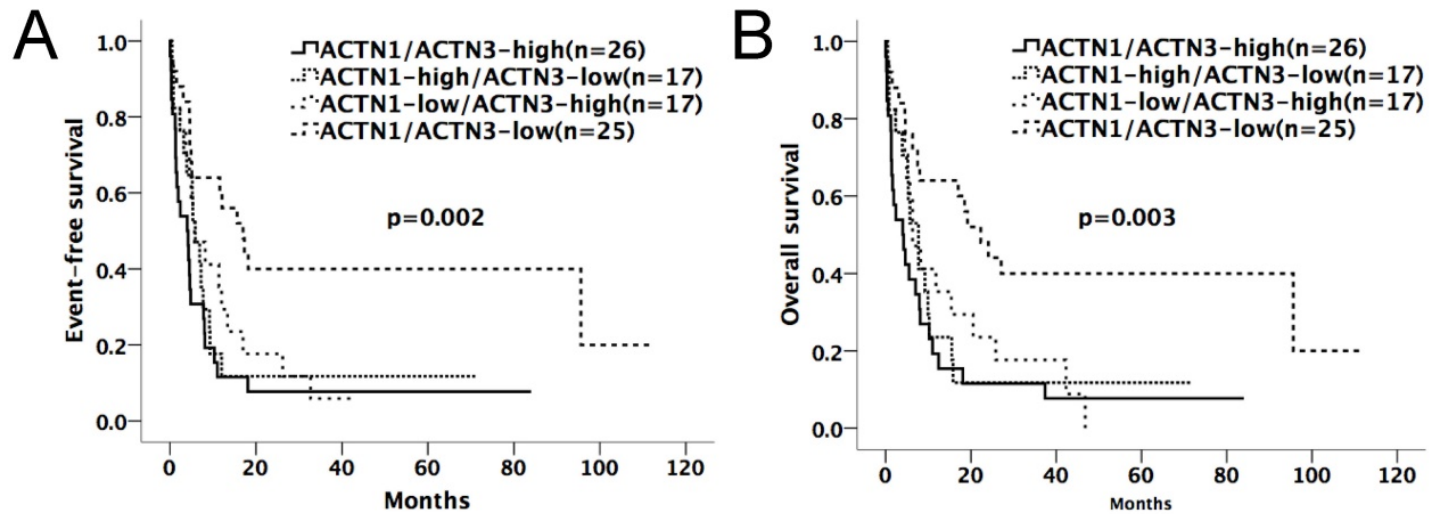

Figure 2. Kaplan-Meier survival curves of different expression levels of ACTNI/ACTN3 in the chemotherapy-only group. Patients with high expressions of either ACTNI or ACTN3 had shorter EFS and OS compared with those with low expressions of both ( $p=0.002$ for EFS, $p=0.003$ for OS, Figure $2 A$ and $2 B$ ).
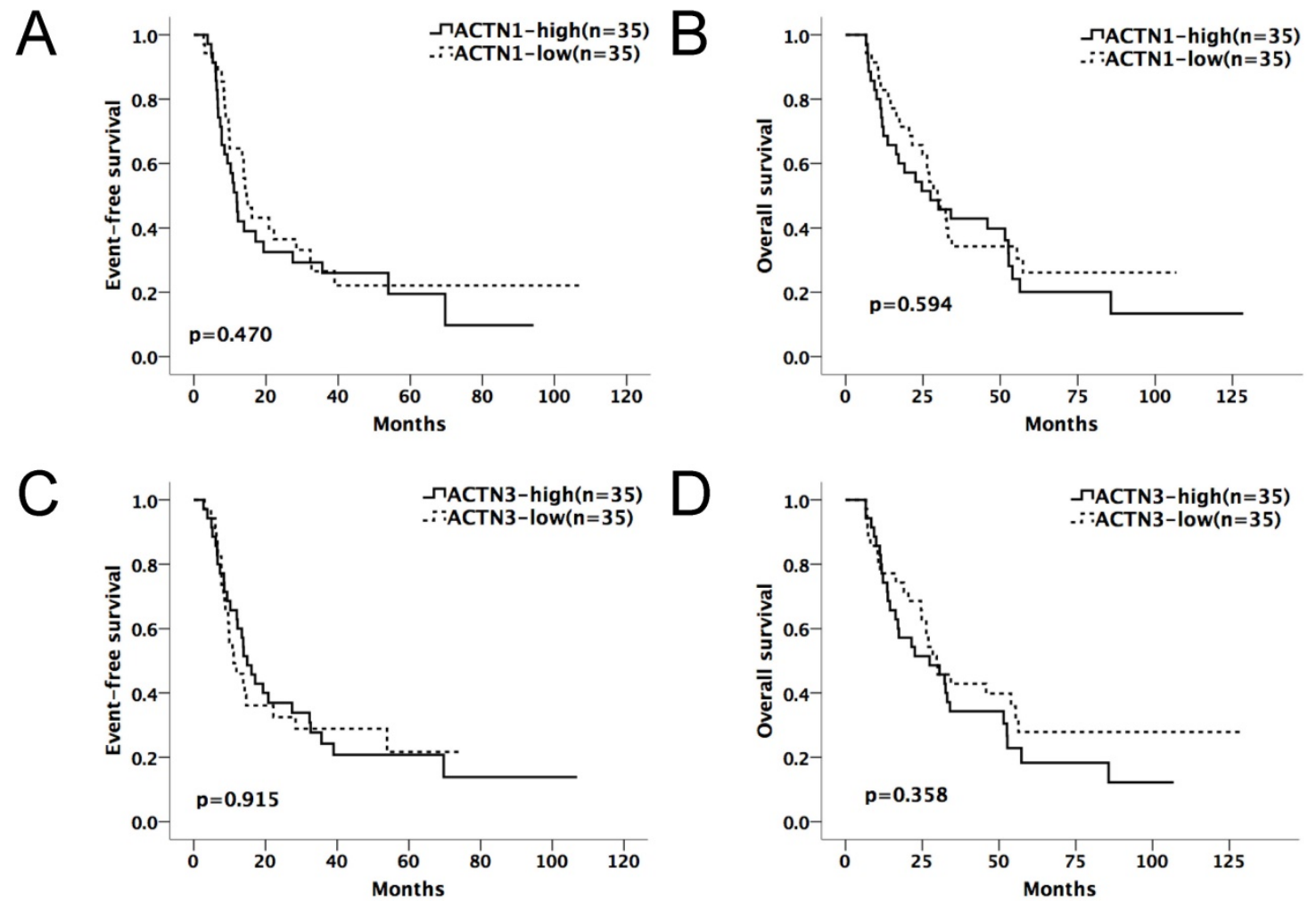

Figure 3. Expression levels of ACTNI/ACTN3 and patients' outcome in the allo-HSCT group. No significant difference was found in EFS or OS comparing high and low expression groups of $A C T N I$ and $A C T N 3$. (p $>0.05$, Figure $3 \mathrm{~A}$ and $3 B$ ). 
Table 3. Multivariate analysis for EFS and OS in the chemotherapy-only group

\begin{tabular}{|c|c|c|c|c|}
\hline \multirow[t]{2}{*}{ Variables } & \multicolumn{2}{|l|}{ EFS } & \multicolumn{2}{|l|}{ OS } \\
\hline & HR $(95 \% \mathrm{CI})$ & $P$-value & $\mathrm{HR}(95 \% \mathrm{CI})$ & $P$-value \\
\hline ACTN1 (high vs. low) & $2.310(1.257-4.245)$ & 0.007 & $2.020(1.110-3.673)$ & 0.021 \\
\hline ACTN2 (high vs. low) & $1.679(0.976-2.890)$ & 0.061 & $1.519(0.887-2.601)$ & 0.128 \\
\hline ACTN3 (high vs. low) & $1.800(1.005-3.225)$ & 0.048 & $2.067(1.134-3.768)$ & 0.018 \\
\hline Age ( $\geq 60$ vs. $<60$ years) & $3.329(1.732-6.400)$ & 0.000 & $2.819(1.486-5.348)$ & 0.002 \\
\hline WBC ( $\geq 15$ vs. $\left.<15 \times 10^{9} / \mathrm{L}\right)$ & $1.701(0.951-3.040)$ & 0.073 & $1.590(0.903-2.800)$ & 0.108 \\
\hline FLT3-ITD (positive vs. negative) & $1.016(0.505-2.043)$ & 0.964 & $1.000(0.485-2.061)$ & 1.000 \\
\hline NPM1 (mutated vs. wild) & $1.293(0.630-2.651)$ & 0.484 & $0.948(0.470-1.912)$ & 0.882 \\
\hline DNMT3A (mutated vs. wild) & $1.507(0.795-2.857)$ & 0.209 & $1.786(0.946-3.372)$ & 0.073 \\
\hline IDH1/2 (mutated vs. wild) & $0.507(0.244-1.051)$ & 0.068 & $0.526(0.257-1.076)$ & 0.079 \\
\hline RUNX1 (mutated vs. wild) & $2.353(0.903-6.134)$ & 0.080 & $2.446(0.942-6.347)$ & 0.066 \\
\hline TET2 (mutated vs. wild) & $0.542(0.240-1.225)$ & 0.141 & $0.363(0.155-0.846)$ & 0.019 \\
\hline NRAS/KRAS (mutated vs. wild) & $0.663(0.301-1.461)$ & 0.308 & $0.812(0.373-1.768)$ & 0.600 \\
\hline
\end{tabular}

Abbreviations: EFS, Event-free survival; OS, Overall survival; WBC, white blood cell; HR, hazard ratio; CI, confidence interval.

\section{Discussion}

Since Honda, et. al., 7 found that ACTN4 was associated with poor prognosis in breast cancer patients, much laboratory and clinical work has been done to illustrate the relationship between actinins and solidary tumors, but little attention had been paid to that between actinins and hematological disorders. Our study was among the first to glimpse into the prognostic significance of ACTN1-4 in AML patients. High expression levels of ACTN1 and ACTN3 acted as adverse factors for outcome in the chemotherapy-only group. However, in the allo-HSCT group, we did not observe any effect of ACTN1 and ACTN3 on the patients' survival.

ACTN4 has been wildly studied for its function in tumors. Like ACTN4, ACTN1 is expressed in most cell types. Given the many similarities in amino acid sequence and actin-binding properties, it would be possible for different ACTNs to have overlapping functions. ${ }^{6}$ ACTN1 participates in the assembly of F-actin at invadopodia, modulates cell adhesion through regulation of focal adhesion kinase-Src interaction ${ }^{14,15}$. Increased level of ACTN1 in the cell promotes migration and loss of polarity by reorganizing the actin cytoskeleton and E-cadherin-based adhesions" ${ }^{16}$. ACTN3 is the "speed gene" that determines the performances of athletes, with $18 \%$ of the human population totally deficient in this gene ${ }^{17}$, but its functions in diseases have rarely been studied. The knocking-off of ACTN3 in mouse muscle can increase the activity of aerobic metabolism and influence sarcomere composition in a dose-dependent fashion ${ }^{18}$. The oncogenic effect of ACTN1 and the metabolic effect of ACTN3 could help explain the findings in our study, though the clear pathophysiological mechanism requires careful laboratory experiments to further delineate.

As the role of actinins in cancer progression became clear in recent years, targeted treatment against actinins has been under development. Craig, et. al., used small interfering RNAs to reduce the expression of ACTN1 in murine tumor cells. ACTN1 silencing disrupted cancer cell adhesion to murine surgical wounds and thereby prolonged the tumor-free survival ${ }^{19}$. Previous studies also reported that transforming growth factor beta (TGF- $\beta$ ) could induce ACTN1 mRNA expression, so targeting TGF- $\beta$ would be an alternative way to suppress ACTN ${ }^{20,21}$. Our study pointed out that ACTN1 and ACTN3 might also be potential targets in AML treatment.

High ACTN1/3 expression was associated with some of the traditional AML risk factors, such as older age, complex karyotype, and poor-risk cytogenetics. Despite the associations, ACTN1/3 expression independently contributed to the poor prognosis in AML patients receiving only chemotherapy. This highlights the fact that although AML prognostication is complex and many factors are intertwined, a single gene could still exert a strong impact.

Allo-HSCT is a powerful treatment of AML to and it can overcome the harmful effect of some high-risk molecular biomarkers ${ }^{22}$. In our study, the adverse effect of high expression levels of ACTN1 and ACTN3 was not observed in the allo-HSCT group, suggesting that allo-HSCT might surmount the adverse effect of ACTN1 and ACTN3 overexpression in AML patients.

\section{Conclusion}

In conclusion, our study found that high expression levels of ACTN1 and ACTN3 at diagnosis indicated unfavorable outcome in AML patients. The pathophysiological mechanism behind this remained to be elucidated. In the future, ACTN1 and ACTN3 could be considered used as biomarkers and indicators for allo-HSCT in AML, if their significant prognostic value were to be confirmed in larger prospective cohorts.

\section{Abbreviations}

ACTN: actinin; ALL: acute lymphoblastic leukemia; AML: acute myeloid leukemia; CR: complete remission; EFS: event-free survival; HSCT: 
hematopoietic stem cell transplantation; ICAMs: intercellular adhesion molecules; NGS: next generation sequencing; OS: overall survival; PI3K: phosphoinositide 3- kinase.

\section{Acknowledgements}

This work was supported by grants from the National Natural Science Foundation of China (81500118, 61501519), the China Postdoctoral Science Foundation funded project (project No.2016M600443) and PLAGH project of Medical Big Data (Project No.2016MBD-025).

\section{Authorship}

Xiaoyan $\mathrm{Ke}$ and Lin $\mathrm{Fu}$ designed and oversaw the study; Xinrui Yang wrote the manuscript; Xinrui Yang, Yifan Pang, Jilei Zhang, Jinlong Shi, Xinpei Zhang, Gaoqi Zhang, Siyuan Yang, Jing Wang, Kai $\mathrm{Hu}$, Jijun Wang and Hongmei Jing performed statistical analyses. All authors contributed to data analysis, drafting and revising the paper and agree to be accountable for all aspects of the work.

\section{Ethics Committee Approval and Patient Consent}

Written informed consent was obtained from all patients for the TCGA database, which was approved by the Human Research Ethics Committee of Washington University.

\section{Competing Interests}

The authors have declared that no competing interest exists.

\section{References}

1. Dohner H, Weisdorf DJ, Bloomfield CD. Acute Myeloid Leukemia. N Engl J Med. 2015;373(12):1136-1152.

2. Thol F, Damm F, Ludeking A, et al. Incidence and prognostic influence of DNMT3A mutations in acute myeloid leukemia. J Clin Oncol. 2011:29(21):2889-2896.

3. Mendler JH, Maharry $\mathrm{K}$, Radmacher MD, et al. RUNX1 mutations are associated with poor outcome in younger and older patients with cytogenetically normal acute myeloid leukemia and with distinct gene and MicroRNA expression signatures. J Clin Oncol. 2012;30(25):3109-3118.

4. Yohe S. Molecular Genetic Markers in Acute Myeloid Leukemia. J Clin Med. 2015;4(3):460-478.

5. Murphy AC, Young PW. The actinin family of actin cross-linking proteins - a genetic perspective. Cell Biosci. 2015;5:49.

6. Foley KS, Young PW. The non-muscle functions of actinins: an update. Biochem J. 2014;459(1):1-13

7. Honda K, Yamada T, Endo R, et al. Actinin-4, a novel actin-bundling protein associated with cell motility and cancer invasion. J Cell Biol. 1998;140(6):1383-1393.

8. Yamamoto $\mathrm{S}$, Tsuda $\mathrm{H}$, Honda $\mathrm{K}$, et al. Actinin-4 expression in ovarian cancer: a novel prognostic indicator independent of clinical stage and histological type. Mod Pathol. 2007;20(12):1278-1285.

9. Honda K, Yamada T, Hayashida Y, et al. Actinin-4 increases cell motility and promotes lymph node metastasis of colorectal cancer. Gastroenterology. 2005;128(1):51-62.

10. Burmeister T, Meyer C, Schwartz S, et al. The MLL recombinome of adult CD10-negative B-cell precursor acute lymphoblastic leukemia: results from the GMALL study group. Blood. 2009;113(17):4011-4015.

11. Sen S, Dong M, Kumar S. Isoform-specific contributions of alpha-actinin to glioma cell mechanobiology. Plos One. 2009:4(12):e8427.

12. Hirooka S, Akashi $\mathrm{T}$, Ando $\mathrm{N}$, et al. Localization of the invadopodia-related proteins actinin- 1 and cortactin to matrix-contact-side cytoplasm of cancer cells in surgically resected lung adenocarcinomas. Pathobiology. 2011;78(1):10-23.

13. Agarwal SK, Simonds WF, Marx SJ. The parafibromin tumor suppressor protein interacts with actin-binding proteins actinin-2 and actinin-3. Mol Cancer. 2008;7:65.

14. Yamaguchi H, Ito $\mathrm{Y}$, Miura N, et al. Actinin-1 and actinin-4 play essential but distinct roles in invadopodia formation by carcinoma cells. Eur J Cell Biol. 2017;96(7):685-694

15. Craig DH, Haimovich B, Basson MD. Alpha-actinin-1 phosphorylation modulates pressure-induced colon cancer cell adhesion through regulation of focal adhesion kinase-Src interaction. Am J Physiol Cell Physiol. 2007;293(6):C1862-C1874

16. Kovac B, Makela TP, Vallenius T. Increased alpha-actinin-1 destabilizes E-cadherin-based adhesions and associates with poor prognosis in basal-like breast cancer. Plos One. 2018;13(5):e196986.

17. MacArthur DG, North KN. A gene for speed? The evolution and function of alpha-actinin-3. Bioessays. 2004;26(7):786-795.

18. MacArthur DG, Seto JT, Chan S, et al. An Actn3 knockout mouse provides mechanistic insights into the association between alpha-actinin-3 deficiency and human athletic performance. Hum Mol Genet. 2008;17(8):1076-1086.

19. Craig DH, Downey C, Basson MD. SiRNA-mediated reduction of alpha-actinin-1 inhibits pressure-induced murine tumor cell wound implantation and enhances tumor-free survival. Neoplasia. 2008;10(3):217-222.

20. Bakin AV, Safina A, Rinehart C, Daroqui C, Darbary H, Helfman DM. A critical role of tropomyosins in TGF-beta regulation of the actin cytoskeleton and cell motility in epithelial cells. Mol Biol Cell. 2004;15(10):4682-4694.

21. Xie L, Law BK, Aakre ME, et al. Transforming growth factor beta-regulated gene expression in a mouse mammary gland epithelial cell line. Breast Cancer Res. 2003;5(6):R187-R198

22. Gupta V, Tallman MS, Weisdorf DJ. Allogeneic hematopoietic cell transplantation for adults with acute myeloid leukemia: myths, controversies, and unknowns. Blood. 2011;117(8):2307-2318. 